\title{
Cosmological parameters from strong gravitational lensing and stellar dynamics in elliptical galaxies
}

\author{
C. Grillo ${ }^{1,2}$, M. Lombardi ${ }^{1,2}$, and G. Bertin ${ }^{2}$ \\ ${ }^{1}$ European Southern Observatory, Karl-Schwarzschild-Str. 2, 85748 Garching bei München, Germany \\ e-mail: cgrillo@eso.org \\ 2 Università degli Studi di Milano, Department of Physics, via Celoria 16, 20133 Milan, Italy
}

Received 23 March 2007 / Accepted 26 October 2007

\begin{abstract}
Context. Observations of the cosmic microwave background, light element abundances, large-scale distribution of galaxies, and distant supernovae are the primary tools for determining the cosmological parameters that define the global structure of the Universe. Aims. Here we illustrate how the combination of observations related to strong gravitational lensing and stellar dynamics in elliptical galaxies offers a simple and promising way to measure the cosmological matter and dark-energy density parameters.

Methods. A gravitational lensing estimate of the mass enclosed inside the Einstein circle can be obtained by measuring the Einstein angle, once the critical density of the system is known. A model-dependent dynamical estimate of this mass can also be obtained by measuring the central velocity dispersion of the stellar component. By assuming the well-tested homologous $1 / r^{2}$ (isothermal) profile for the total (luminous+dark) density distribution in elliptical galaxies acting as lenses, these two mass measurements can be properly compared. Thus, a relation between the Einstein angle and the central stellar velocity dispersion is derived, and the cosmological matter and the dark-energy density parameters can be estimated from this.

Results. We determined the accuracy of the cosmological parameter estimates by means of simulations that include realistic measurement uncertainties on the relevant quantities. Interestingly, the expected constraints on the cosmological parameter plane are complementary to those coming from other observational techniques. Then, we applied the method to the recent data sets of the Sloan Lens ACS (SLACS) and the Lenses Structure and Dynamics (LSD) Surveys, and showed that the concordance value between 0.7 and 0.8 for the dark-energy density parameter is included in our $99 \%$ confidence regions.

Conclusions. The small number of lenses available to date prevents us from precisely determining the cosmological parameters, but it still proves the feasibility of the method. When applied to samples made of hundreds of lenses that are expected to become available from forthcoming deep and wide surveys, this technique will be an important alternative tool for measuring the geometry of the Universe.
\end{abstract}

Key words. cosmology: theory - cosmology: observations - galaxies: distances and redshifts - galaxies: kinematics and dynamics galaxies: elliptical and lenticular, $\mathrm{cD}$ - gravitational lensing

\section{Introduction}

The Universe appears to be dominated by dark-energy and dark matter. Although the physical nature of these dark components is still unknown, the standard cosmological $\Lambda C D M$ model with only a few parameters fits most of the current data well: precision measurements of the anisotropies in the cosmic microwave background ( $C M B$; Bennett et al. 2003; Spergel et al. 2003, 2007), the observed abundances of light elements (Burles et al. 2001; Cyburt et al. 2003), the large-scale distribution of galaxies (LSS; Tegmark et al. 2004; Cole et al. 2005), and the luminositydistance relationship for distant type Ia supernovae (SNIa; Riess et al. 1998, 2004; Perlmutter et al. 1999). In this standard model, the Universe is homogeneous and isotropic on its largest scales, and its geometry appears to be flat $\left(\Omega=\Omega_{\mathrm{m}}+\Omega_{\Lambda} \approx 1\right)$; the total mass-energy density is mainly in the form of darkenergy $\left(\Omega_{\Lambda} \approx 0.7\right)$ and matter $\left(\Omega_{\mathrm{m}} \approx 0.3\right)$, ordinary and dark. These values of the cosmological parameters imply a fairly recent transition from a decelerating to an accelerating universal expansion. The Hubble Space Telescope (HST) Key Project has measured the current expansion rate, the Hubble parameter $H_{0}=(72 \pm 8) \mathrm{km} \mathrm{s}^{-1} \mathrm{Mpc}^{-1}$ (Freedman et al. 2001). The estimates of different cosmological parameters from a single observational method are often correlated (hence "degenerate") and exhibit significant uncertainties. For instance, from the WMAP three year data alone, without a prior on the flatness of the Universe, the best-fit model is characterized by $\Omega_{\mathrm{m}}=0.42$, $\Omega_{\Lambda}=0.63, H_{0}=55 \mathrm{~km} \mathrm{~s}^{-1} \mathrm{Mpc}^{-1}$ (Spergel et al. 2007), values that are quite different from the concordance values reported above. This suggests that precise measurements of the cosmological parameters can only be obtained by using complementary techniques. In fact, considerable efforts are still being made in order to secure accurate measurements of these parameters (in particular, see the scientific goals of the forthcoming PLANCK and SNAP missions).

The deflection of light due to gravitational lensing is sensitive to the total matter density of the structures in the Universe, independently of the nature or dynamical state of the deflecting mass. Therefore, strong and weak gravitational lensing provide valuable tools for measuring the distribution of mass. In particular, cosmic shear estimates of the amplitude of the weak lensing distorsions of distant sources over a wide range of angular scales (Bartelmann \& Schneider 1999) are a very encouraging way to study the large-scale structure of the Universe, and therefore to probe the parameters that define the relevant cosmological model (Refregier 2003). Further constraints on the 
geometry of the Universe can also be provided by the abundance of lenses or arcs observed in lens surveys (see Bartelmann \& Weiss 1994, for a numerical approach; Mitchell et al. 2005, for observational results from the CLASS Survey described by Myers et al. 2003; and Browne et al. 2003) and by strong (Link \& Pierce 1998; Soucail et al. 2004) or weak (Lombardi \& Bertin 1999; Jain \& Taylor 2003) lensing mass reconstructions in clusters of galaxies. Isolated strong lens galaxies offer another possibility to measure the cosmological parameters (Kochanek 1992, 1996; Myungshin et al. 1997), including the Hubble parameter (Refsdal 1964; Koopmans et al. 2003b; Mörtsell \& Sunesson 2006).

The various techniques that have been proposed are limited by several assumptions. For example, a measurement of the matter density parameter from cosmic shear is degenerate with that of the normalization of the amplitude of the power spectrum of matter perturbations $\left(\sigma_{8}\right)$; moreover, an extremely large number of high-quality galaxy images and some modeling on the growth of the structure in the Universe are required. For techniques based on gravitational lens statistics, the luminosity function, the relation between luminosity and velocity dispersion, and the density profile of the lens galaxies play an important role. The use of arcs statistics awaits more realistic simulations of clusters and observations, since different studies have led to contrasting results (e.g., Bartelmann et al. 1998; Dalal et al. 2004). The estimates of the cosmological parameters from cluster mass reconstructions have also two distinct limitations: the presence of possible substructure in the region of multiple image formation (for strong lensing) and the need for a high density of background galaxies (for weak lensing).

In this paper we propose a new technique that, starting from strong gravitational lensing and stellar dynamics observations in elliptical galaxies, is able to probe the geometry of the Universe in a different and effective way. The paper is organized as follows. In Sect. 2, we describe our method to estimate the matter and dark-energy density parameters. Then, in Sect. 3, we determine through simulations the precision attainable in these measurements of the cosmological parameters. Good estimators for the quantities relevant to the problem are identified in Sect. 4. In Sect. 5, we apply our technique to the data collected and just published of two surveys of lens galaxies for which stellar dynamical measurements are available. Finally, in Sect. 6, we summarize and discuss the results obtained.

\section{The method}

It is known that in an axisymmetric lens multiple images can only form in the vicinity of the so-called Einstein ring, at an angle $\theta_{\mathrm{E}}$ from the center of the lens (see Schneider et al. 1992). From the theory of gravitational lensing, the "mass" $\mathscr{M}_{\text {grl }}$ enclosed within the disk defined by the Einstein ring is directly related to the geometry of the configuration, through the definition of the critical density $\left(\mathscr{M}_{\text {grl }}=\Sigma_{\text {cr }} \pi \theta_{\mathrm{E}}^{2}\right)$. This "mass" $\mathscr{M}_{\text {grl }}$ is connected to the intrinsic mass $M_{\mathrm{grl}}$ of the lens by the distance to the lens (by converting the Einstein angle into an Einstein radius).

A dynamical estimate $\mathscr{M}_{\text {dyn }}$ of the mass can also be given by measuring the quantity $\sigma_{0}^{2} \theta_{\mathrm{E}}$, where $\sigma_{0}$ is the central velocity dispersion of the stellar component, usually referred to the disk of radius $R_{\mathrm{e}} / 8$ ( $R_{\mathrm{e}}$ being the standard optical effective radius); the dynamical mass is then obtained by multiplication by a suitable factor $\left(\mathscr{M}_{\text {dyn }}=\alpha \sigma_{0}^{2} \theta_{\mathrm{E}}\right)$ that is model-dependent (the lens usually includes a significant dark matter component). Again, in order to relate $\mathscr{M}_{\text {dyn }}$ to the intrinsic mass $M_{\text {dyn }}$ we should convert $\theta_{\mathrm{E}}$ into a radius.
With no need to refer to intrinsic masses (and thus with no need to know the exact distance to the lens, which would bring in a knowledge of the Hubble constant $H_{0}$ ), we thus see that, if we identify $\mathscr{M}_{\text {grl }}=\mathscr{M}_{\text {dyn }}$, the combination of a measurement of $\theta_{\mathrm{E}}$ and of $\sigma_{0}$ should be uniquely related, in the standard cosmological model, to a function of the redshifts $z_{1}$ and $z_{\mathrm{s}}$ (of the lens and of the source, respectively) and of the cosmological parameters $\Omega_{\mathrm{m}}$ and $\Omega_{\Lambda}$. Note that, in this context, $z_{1}$ and $z_{\mathrm{s}}$ can be considered to be measured with negligible errors.

Several studies, based on various dynamical tracers (stars, globular clusters, planetary nebulae, X-ray halos, HI disks and rings), have established that bright elliptical galaxies of the local universe, as a rule, exhibit approximately flat circular velocity curves (e.g., Gerhard et al. 2001; Peng et al. 2004), thus suggesting that the structure of these systems should be considered as approximately homologous, with the total density distribution (luminous+dark) close to that of a singular isothermal sphere (SIS; $\rho \propto 1 / r^{2}$ ). These detailed dynamical studies refer to nearby galaxies and thus the result obtained does not depend on the values of the cosmological parameters. Of course, this is a zeroth-order description, and different galaxies may exhibit different deviations from this "universal" total density profile.

A number of investigations of galaxies at cosmologically significant distances address observed properties that result from the combined effects of evolution and of the geometry of the universe. The interpretation of these data can thus be obtained in different ways. For example, in the study of the Fundamental Plane out to $z \approx 1$ (e.g., see Treu et al. 2002, and other parallel investigations, many of which quoted there), one may assume approximate structural homology and a given cosmological model and use the data on the observed change in the Fundamental Plane to derive information on the evolution properties of the observed stellar populations.

Here we recall that, under the assumption of the concordance cosmological model $\left(\Omega_{\mathrm{m}}=0.3, \Omega_{\Lambda}=0.7, H_{0}=\right.$ $70 \mathrm{~km} \mathrm{~s}^{-1} \mathrm{Mpc}^{-1}$ ), analyses of strong gravitational lensing alone (e.g., Rusin et al. 2003) or combined measurements of stellar dynamics and gravitational lensing (e.g., Treu \& Koopmans 2004; Koopmans et al. 2006) have confirmed the persistence of structural homology, i.e., that little evolution appears to take place in the observed total density profile of bright ellipticals, in the sense that they are found to be characterized by approximately SIS density profiles also at cosmologically significant distances. In Sect. 5 we will show that this conclusion is robust with respect to the choice of the adopted cosmological parameters.

These findings have encouraged us to explore the consequences of considering the combined measurements of stellar dynamics and gravitational lensing on distant ellipticals, starting from the simplifying assumption that homology is indeed strictly followed by these systems; in particular, we wish to explore whether this assumption, applied to the interpretation of the data, may lead to interesting constraints on the values of the parameters that define the geometry of the universe. In other words, we wish to check the consequences on the cosmological parameters of assuming from the very beginning that (1) the espression for $\mathscr{M}_{\text {grl }}$ is basically that associated with an SIS, and (2) no significant variation on the "virial coefficient" $\alpha$ is present from galaxy to galaxy.

In practice, we proceed as follows. We note that for an SIS

$\theta_{\mathrm{E}}=4 \pi\left(\frac{\sigma_{\mathrm{SIS}}}{c}\right)^{2} \frac{D_{\mathrm{ls}}}{D_{\mathrm{os}}}$,

where $\sigma_{\text {SIS }}$ is the lens "velocity dispersion", $c$ is the light speed, $D_{\mathrm{ls}}$ and $D_{\mathrm{os}}$ are the lens-source and the observer-source 
angular diameter distances, respectively. We then bypass the issues raised by dynamical modeling by recalling that $\sigma_{0}$ turns out to be a good estimate of $\sigma_{\text {SIS }}$. This latter point, exploited by Kochanek (1993, 1994), was confirmed by Treu et al. (2006), and is now checked by us independently, by a test described separately in Sect. 4.2 on a sample of eight well-studied nearby ellipticals. Therefore we consider the quantity

$\frac{c^{2}}{4 \pi} \frac{\theta_{\mathrm{E}}}{\sigma_{0}^{2}}=\frac{D_{\mathrm{ls}}}{D_{\mathrm{os}}}=r\left(z_{\mathrm{l}}, z_{\mathrm{s}} ; \Omega_{\mathrm{m}}, \Omega_{\Lambda}\right)$,

as the observable that will be used to produce, by studying a statistically significant sample of lenses, a measurement of $\Omega_{\mathrm{m}}$ and $\Omega_{\Lambda}$.

Given the weak dependence of the relevant coefficients, such as $\alpha$, on the detailed relative distributions of dark and luminous matter in galaxies, the method is expected to be robust. In particular, the entire argument could be easily generalized to non-axisymmetric lenses by referring to the properties of the socalled singular isothermal ellipsoid (see Kormann et al. 1994).

Figure 1 illustrates the distance ratio $r$ of Eq. (2) (which does not have a simple analytic form) versus the source redshift, as a function of the lens redshift and of the matter and dark-energy density parameters. In general, different cosmological models give values of $r$ which differ more clearly at higher values of the source redshift (see the last three panels); in addition, the quantity $r$ is more sensitive to small variations of $\Omega_{\Lambda}$ than of $\Omega_{\mathrm{m}}$ (compare the second and third panels). As a consequence, we naturally expect this method to be optimally efficient in measuring $\Omega_{\Lambda}$, provided we have at our disposal a sample of lenses at sufficiently high redshifts.

\section{Simulated measurements of the cosmological parameters}

In order to explore the precision with which the above technique can probe the cosmological parameters, we have performed several simulations. We modelled each lens as a singular isothermal sphere with an external shear component. Because of the known degeneracy between external shear and ellipticity (Witt \& Mao 1997), we remark that our modeling choice is also good at describing simulations with singular isothermal ellipsoid models. As suggested by real lensing systems, we considered the lens redshift uniformly distributed between 0 and 1 and the source redshift between 1 and 3.5; the lens velocity dispersion and the external shear values were drawn from uniform distributions ranging from 100 to $350 \mathrm{~km} \mathrm{~s}^{-1}$ for the first, from 0 to 0.2 in magnitude and from $0^{\circ}$ to $180^{\circ}$ in orientation for the second (the role of the external shear component will become relevant in Sect. 4.1). We calculated the Einstein angles from Eq. (1) in a $\left(\Omega_{\mathrm{m}}, \Omega_{\Lambda}\right)=(0.3,0.7)$ cosmology and completed each lensing system simulating randomly, inside a square of side $\theta_{\mathrm{E}}$ and centered on the lens, the position of a source. Only the lenses with two images of this source and with $\theta_{\mathrm{E}}$ greater than $0.5^{\prime \prime}$ were accepted for the next analyses. The reason is that we wanted to investigate multiple image systems similar to those observed, where the images are far enough from the center of the galaxy acting as a lens to be resolved with the present technology.

We started by examining the dependence of the error estimates on $\Omega_{\mathrm{m}}$ and $\Omega_{\Lambda}$ on the simulated uncertainties on the Einstein angle and on the central velocity dispersion. In order to do this, we minimized a chi-square $\left(\chi^{2}\right)$ like estimator with respect to the two cosmological parameters. This function is defined by comparing the "observational" distance ratio calculated
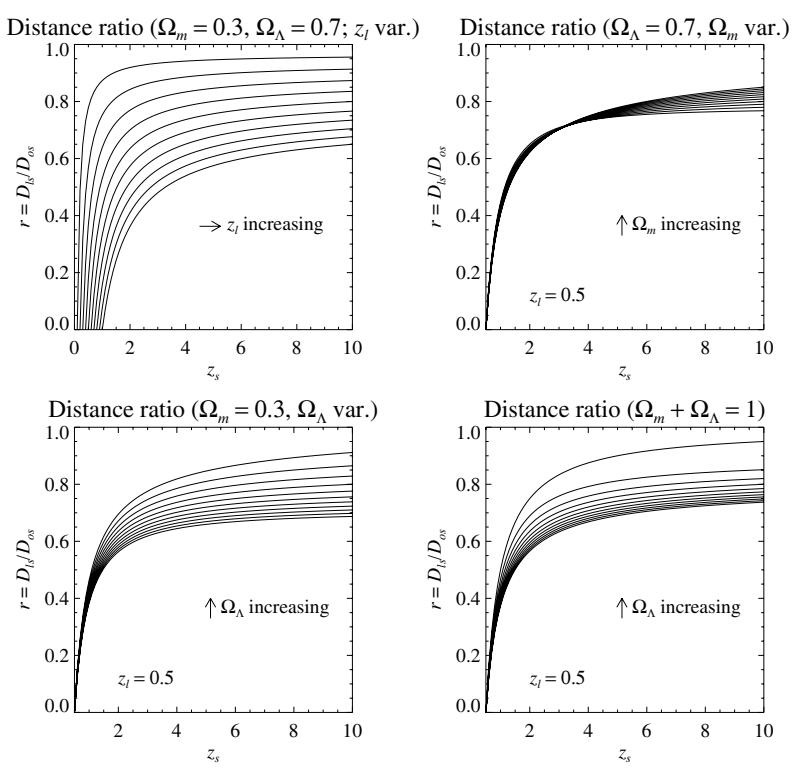

Fig. 1. Dependence of the angular diameter distance ratio $r=D_{\mathrm{ls}} / D_{\mathrm{os}}$ on the lens redshift (top left), matter density parameter (top right), darkenergy density parameter (bottom left), and matter and dark-energy density parameters in a flat cosmological model (bottom right). In the first panel, $\left(\Omega_{\mathrm{m}}, \Omega_{\Lambda}\right)=(0.3,0.7)$ are fixed and $z_{1}$ is varied from 0.1 to 1 , with a regular step of 0.1 . The arrow shows the increasing direction of $z_{\mathrm{l}}$. In the second panel, $\Omega_{\Lambda}=0.7$ and $z_{1}=0.5$ are fixed and $\Omega_{\mathrm{m}}$ is varied from 0 to 1 , with a regular step of 0.1 . The arrow shows the increasing direction of $\Omega_{\mathrm{m}}$. In the third panel, $\Omega_{\mathrm{m}}=0.3$ and $z_{\mathrm{l}}=0.5$ are fixed and $\Omega_{\Lambda}$ is varied from 0 to 1 , with a regular step of 0.1 . The arrow shows the increasing direction of $\Omega_{\Lambda}$. In the fourth panel, $\Omega_{\mathrm{m}}+\Omega_{\Lambda}=1$ and $z_{1}=0.5$ are fixed and $\Omega_{\Lambda}$ is varied from 0 to 1 , with a regular step of 0.1 . The arrow shows the increasing direction of $\Omega_{\Lambda}$.

from $\theta_{\mathrm{E}}$ and $\sigma_{0}$, that is the quantity on the left in Eq. (2), and the "theoretical" distance ratio obtained from the lens and source redshifts, that is the quantity on the right in Eq. (2):

$\chi^{2}\left(\Omega_{\mathrm{m}}, \Omega_{\Lambda}\right)=\sum_{i=1}^{N} \frac{\left(\frac{c^{2}}{4 \pi} \frac{\theta_{\mathrm{E}_{i}}}{\sigma_{0_{i}}^{2}}-r\left(z_{\mathrm{l}_{i}}, z_{\mathrm{s}_{i}} ; \Omega_{\mathrm{m}}, \Omega_{\Lambda}\right)\right)^{2}}{\left(\frac{c^{2}}{4 \pi}\right)^{2}\left[\left(\frac{1}{\sigma_{0_{i}}^{2}}\right)^{2}\left(\delta \theta_{\mathrm{E}_{i}}\right)^{2}+\left(\frac{\theta_{\mathrm{E}_{i}}}{\sigma_{0_{i}}^{4}}\right)^{2}\left(\delta \sigma_{0_{i}}^{2}\right)^{2}\right]}$.

We included realistic measurement errors on the Einstein angle $\left(\delta \theta_{\mathrm{E}}\right)$ and on the central velocity dispersion $\left(\delta \sigma_{0}\right)$, considering normal distributions with standard deviations equal to $4 \%$, $5 \%, 6 \%$, and $7 \%$ of the true values. At first, we performed 2000 minimizations for samples of 100 and 200 lenses, assigning a nominal $0 \%$ uncertainty to one of the two quantities and varying the error on the other in the range reported above. Then, we repeated the analysis just described with the additional hypothesis of a flat cosmological model $\left(\Omega_{\mathrm{m}}+\Omega_{\Lambda}=1\right)$. Finally, we simulated estimates of the cosmological parameters, in general and flat cosmology models, considering 5\% errors on both the Einstein angle and the central velocity dispersion. This is the precision with which these quantities can be measured at present by means of the best ground and space-based telescopes.

The results for the joint and single probability density functions $(f)$ of the cosmological parameters are summarized by Figs. $2-4$ and by Tables $1-3$. As a first good indication, the minimum $\chi^{2}$ was found to be asymptotically unbiased, i.e. centered on the original values $\left(\Omega_{\mathrm{m}}, \Omega_{\Lambda}\right)=(0.3,0.7)$ in the limit of small uncertainties on $\theta_{\mathrm{E}}$ and $\sigma_{0}$. Then, we remark that the true values of the cosmological parameters are always inside the regions and 

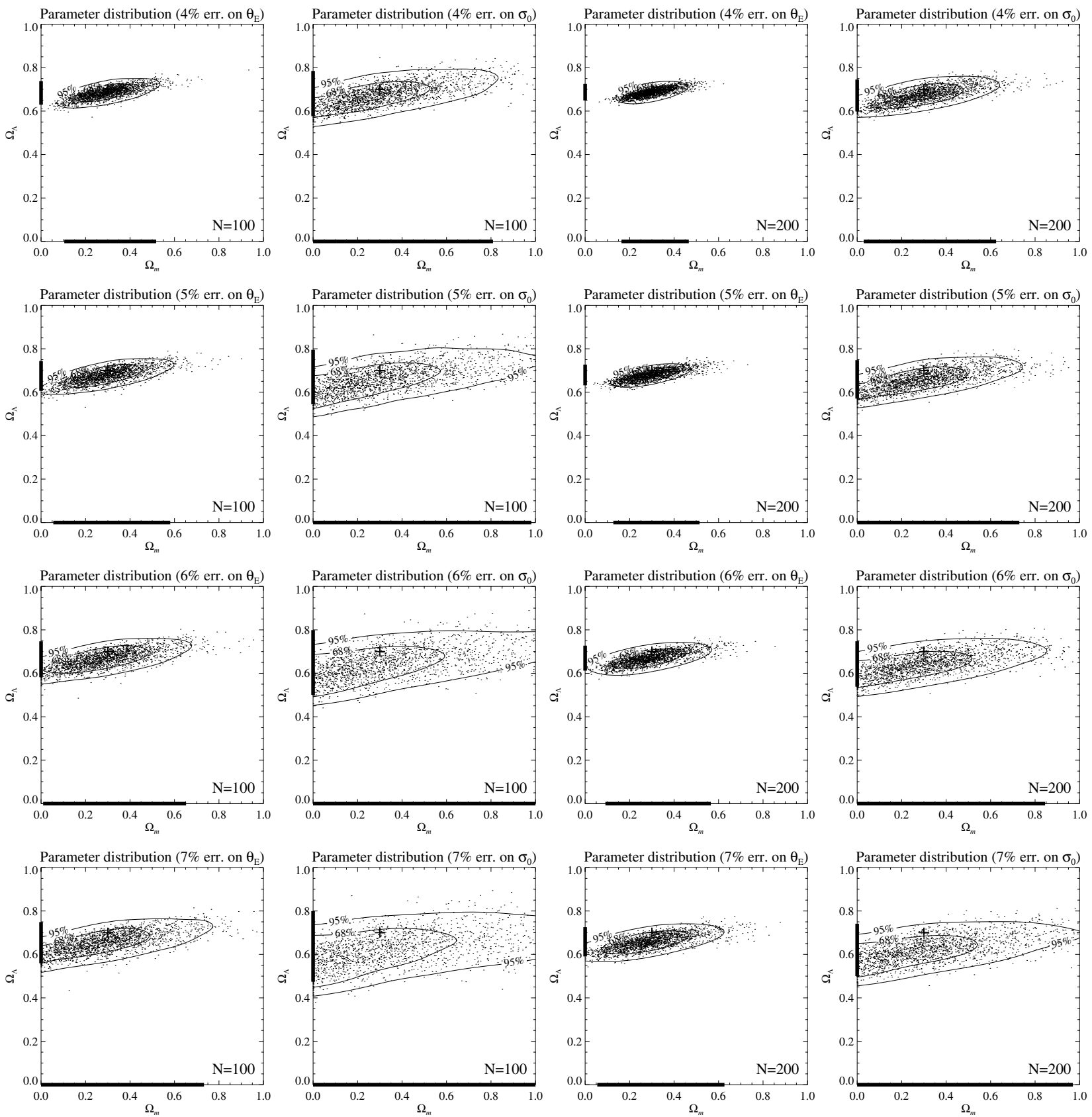

Fig. 2. Estimates of the cosmological parameters. Simulation of 2000 measurements composed of 100 (on the left) and 200 (on the right) lenses each, with different uncertainties (increasing from the top to the bottom) on the Einstein angle (first and third column) and on the central velocity dispersion (second and fourth column). A nominal $0 \%$ uncertainty is assigned to the quantity not mentioned in the panels. Thick bars on the co-ordinate axes and contour levels on the planes represent, respectively, the $95 \%$ confidence intervals and the $68 \%$ and $95 \%$ confidence regions for the cosmological parameters. A cross shows the position of the true parameters: $\left(\Omega_{\mathrm{m}}, \Omega_{\Lambda}\right)=(0.3,0.7)$.

intervals at the $95 \%$ confidence level, but their boundaries are not symmetrical. In fact, in all the three previously mentioned tables these confidence intervals for $\Omega_{\mathrm{m}}$ are more extended on the high side; in contrast, the intervals for $\Omega_{\Lambda}$ are generally larger on the low side. Moreover, since the distance ratio $r$ depends linearly on the Einstein angle and quadratically on the central velocity dispersion, larger confidence regions and intervals are obtained when a fixed uncertainty is considered on the latter quantity. The assumption about the flatness of the Universe makes our technique more powerful, allowing a better estimate of the darkenergy density parameter, as can be seen by comparing Tables 1 and 2. Finally, from the results of Fig. 4 and Table 3, we argue that the current measurement precision already allows a good estimate of $\Omega_{\Lambda}$ from a sufficiently large sample of lenses.

From what discussed so far, we conclude that this method is especially well-suited to measure the dark-energy density parameter. This is not surprising, given the comments made in the previous section about the different dependence of the distance ratio on the two cosmological parameters. In view of the fact that, so far, only supernova observations have been able to reveal directly a significant dark-energy component, our technique offers a promising new test for the concordance model. It is also particularly interesting to notice that the $95 \%$ confidence regions on the parameter planes in Figs. 2 and 4 are oriented in such a 

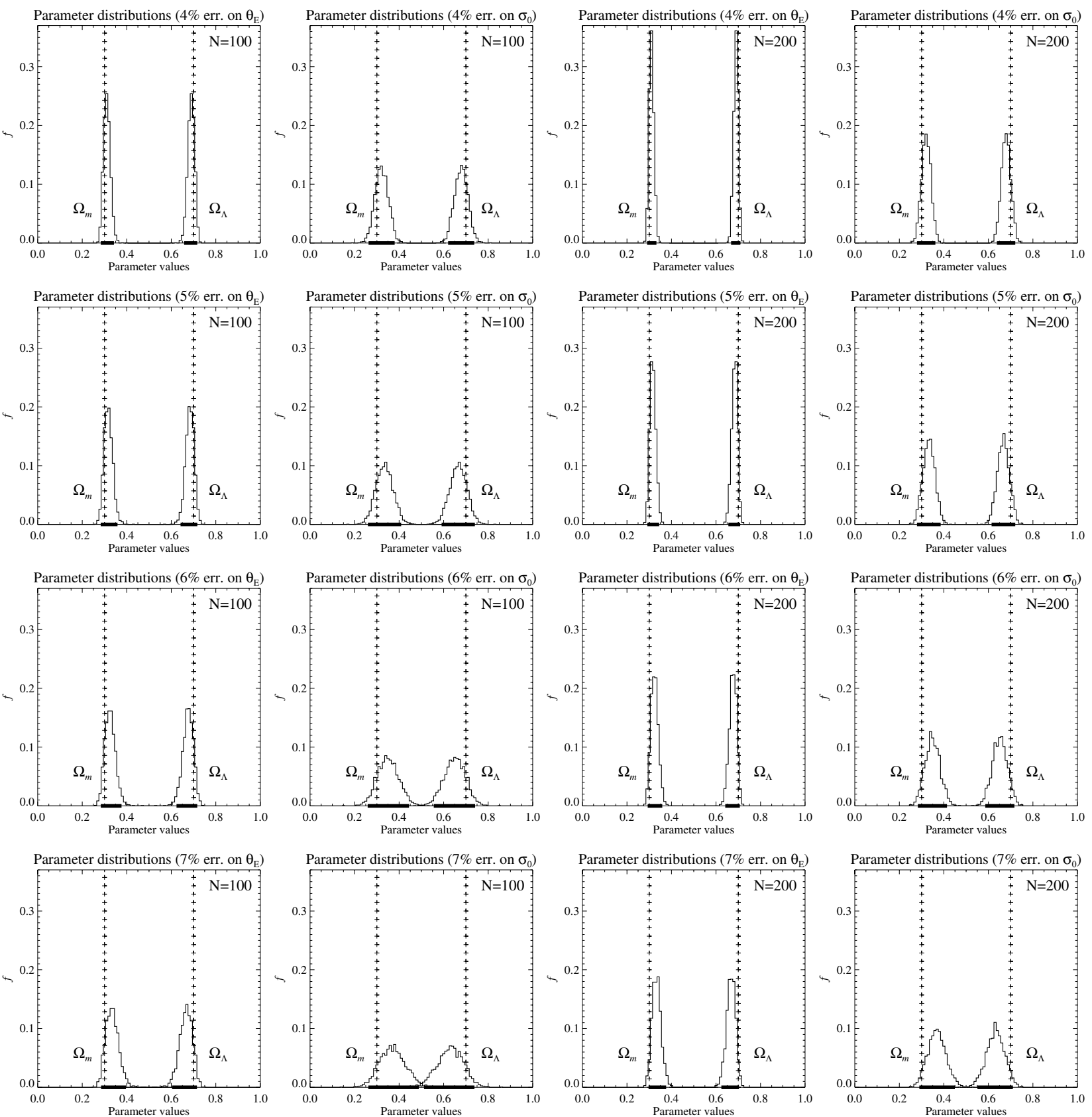

Fig. 3. Estimates of the cosmological parameters in a flat model $\left(\Omega_{\mathrm{m}}+\Omega_{\Lambda}=1\right.$ ). Simulation of 2000 measurements composed of 100 (on the left) and 200 (on the right) lenses each, with different uncertainties (increasing from the top to the bottom) on the Einstein angle (first and third column) and on the central velocity dispersion (second and fourth column). A nominal $0 \%$ uncertainty is assigned to the quantity not mentioned in the panels. Thick bars on the abscissa axes represent the 95\% confidence intervals for the cosmological parameters. Crosses show the position of the true parameters: $\left(\Omega_{\mathrm{m}}, \Omega_{\Lambda}\right)=(0.3,0.7)$.

way to be complementary to the results of the other cosmological probes currently considered.

\section{Diagnostics of the relevant quantities}

\subsection{The Einstein angle}

Isolated lens galaxies which show low ellipticity values in the luminous distribution are expected to be well described by axisymmetric models; on the other hand, non-axisymmetric lens models are required to represent galaxies in groups or clusters and galaxies displaying high ellipticity in the luminosity profile. In the former case, the lens properties are embodied by the so-called Einstein angle; in the latter case, an equivalent Einstein angle can still be considered. However, in both cases the uncertainty with which the Einstein angle can be reconstructed by lensing modeling is on the order of $5 \%$, provided high-quality imaging of the systems is available.

In future cosmological studies we will have to handle data sets from wide and deep sky surveys made of hundreds of lenses. In order to evaluate in a simple way $\theta_{\mathrm{E}}$ for lensing systems with two images of the same source, we may define two estimators. They could be used to perform a preliminary fast analysis of the data, before detailed models with better precision are built. (This problem has not been taken into account in the measurements of the cosmological parameters presented in this paper, because in 
Table 1. Intervals at $95 \%$ confidence level for the matter and the dark-energy density parameters.

\begin{tabular}{cccccccccc}
\hline \hline \multicolumn{3}{c}{$N=100$} & & & & $N=200$ & \\
\hline Err. $\theta_{\mathrm{E}}$ & $4 \%$ & $5 \%$ & $6 \%$ & $7 \%$ & Err. $\theta_{\mathrm{E}}$ & $4 \%$ & $5 \%$ & $6 \%$ & $7 \%$ \\
\hline$\Omega_{\mathrm{m}}$ & {$[0.10,0.52]$} & {$[0.06,0.58]$} & {$[0.01,0.65]$} & {$[0.00,0.73]$} & $\Omega_{\mathrm{m}}$ & {$[0.16,0.47]$} & {$[0.13,0.51]$} & {$[0.09,0.57]$} & {$[0.05,0.63]$} \\
$\Omega_{\Lambda}$ & {$[0.63,0.74]$} & {$[0.61,0.74]$} & {$[0.58,0.75]$} & {$[0.56,0.75]$} & $\Omega_{\Lambda}$ & {$[0.65,0.73]$} & {$[0.63,0.73]$} & {$[0.61,0.73]$} & {$[0.59,0.73]$} \\
\hline Err. $\sigma_{0}$ & $4 \%$ & $5 \%$ & $6 \%$ & $7 \%$ & Err. $\sigma_{0}$ & $4 \%$ & $5 \%$ & $6 \%$ & $7 \%$ \\
\hline$\Omega_{\mathrm{m}}$ & {$[0.00,0.81]$} & {$[0.00,0.98]$} & {$[0.00,1.19]$} & {$[0.00,1.41]$} & $\Omega_{\mathrm{m}}$ & {$[0.03,0.63]$} & {$[0.00,0.73]$} & {$[0.00,0.85]$} & {$[0.00,0.97]$} \\
$\Omega_{\Lambda}$ & {$[0.58,0.78]$} & {$[0.54,0.80]$} & {$[0.50,0.80]$} & {$[0.47,0.80]$} & $\Omega_{\Lambda}$ & {$[0.60,0.75]$} & {$[0.57,0.75]$} & {$[0.54,0.75]$} & {$[0.50,0.74]$} \\
\hline
\end{tabular}

Notes. These intervals are obtained by excluding from the $2000 \chi^{2}$ minimizations the 50 smallest and the 50 largest values for $\Omega_{\mathrm{m}}$ and $\Omega_{\Lambda}$. The simulated measurement uncertainties of the Einstein angle (on the top) and of the central velocity dispersion (on the bottom) range from $4 \%$ to $7 \%$ (a nominal 0\% uncertainty is assigned to one of the two quantities and the error on the other is varied) for samples of 100 (on the left) and 200 (on the right) lenses.

Table 2. Intervals at $95 \%$ confidence level for the matter and the dark-energy density parameters in a flat cosmology $\left(\Omega_{\mathrm{m}}+\Omega_{\Lambda}=1\right)$.

\begin{tabular}{cccccccccr}
\hline \hline \multicolumn{9}{c}{$N=100$} & \multicolumn{6}{c}{$N=200$} \\
\hline Err. $\theta_{\mathrm{E}}$ & $4 \%$ & $5 \%$ & $6 \%$ & $7 \%$ & Err. $\theta_{\mathrm{E}}$ & $4 \%$ & $5 \%$ & $6 \%$ & $7 \%$ \\
\hline$\Omega_{\mathrm{m}}$ & {$[0.28,0.34]$} & {$[0.28,0.36]$} & {$[0.28,0.38]$} & {$[0.28,0.40]$} & $\Omega_{\mathrm{m}}$ & {$[0.29,0.33]$} & {$[0.29,0.34]$} & {$[0.29,0.36]$} & {$[0.30,0.38]$} \\
$\Omega_{\Lambda}$ & {$[0.66,0.72]$} & {$[0.64,0.72]$} & {$[0.62,0.72]$} & {$[0.60,0.72]$} & $\Omega_{\Lambda}$ & {$[0.67,0.71]$} & {$[0.66,0.71]$} & {$[0.64,0.71]$} & {$[0.62,0.70]$} \\
\hline Err. $\sigma_{0}$ & $4 \%$ & $5 \%$ & $6 \%$ & $7 \%$ & Err. $\sigma_{0}$ & $4 \%$ & $5 \%$ & $6 \%$ & $7 \%$ \\
\hline$\Omega_{\mathrm{m}}$ & {$[0.26,0.38]$} & {$[0.26,0.41]$} & {$[0.26,0.44]$} & {$[0.26,0.49]$} & $\Omega_{\mathrm{m}}$ & {$[0.28,0.36]$} & {$[0.28,0.39]$} & {$[0.28,0.41]$} & {$[0.29,0.45]$} \\
$\Omega_{\Lambda}$ & {$[0.62,0.74]$} & {$[0.59,0.74]$} & {$[0.56,0.74]$} & {$[0.51,0.74]$} & $\Omega_{\Lambda}$ & {$[0.64,0.72]$} & {$[0.61,0.72]$} & {$[0.59,0.72]$} & {$[0.55,0.71]$} \\
\hline
\end{tabular}

Notes. These intervals are obtained by excluding from the $2000 \chi^{2}$ minimizations the 50 smallest and the 50 largest values for $\Omega_{\mathrm{m}}$ and $\Omega_{\Lambda}$. The simulated measurement uncertainties of the Einstein angle (on the top) and of the central velocity dispersion (on the bottom) range from $4 \%$ to $7 \%$ (a nominal 0\% uncertainty is assigned to one of the two quantities and the error on the other is varied) for samples of 100 (on the left) and 200 (on the right) lenses.
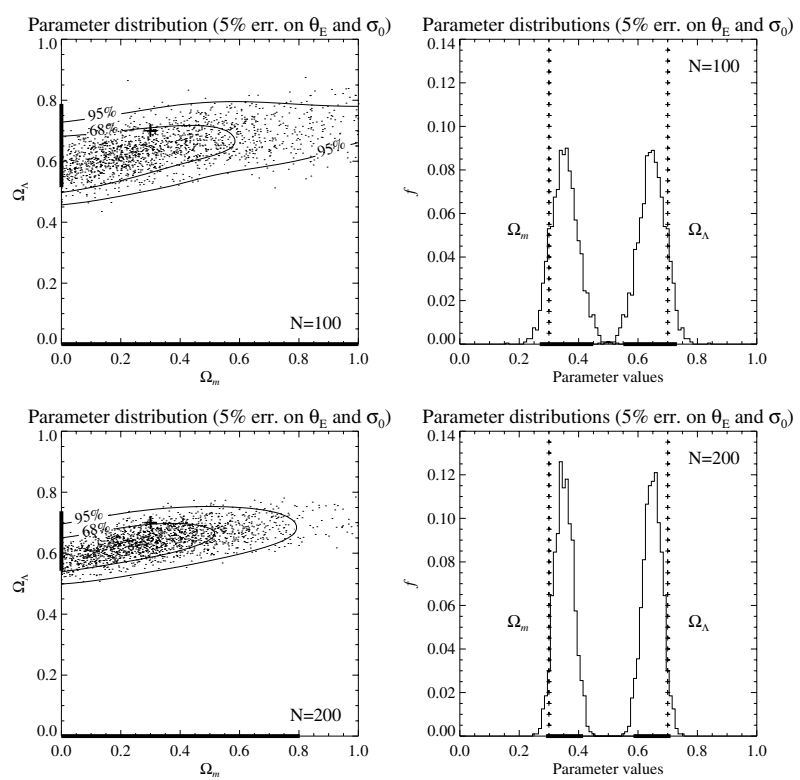

Fig. 4. Estimates of the cosmological parameters assuming $5 \%$ uncertainty on both the Einstein angle and the central velocity dispersion. Simulation of 2000 measurements composed of 100 (on the top) and 200 (on the bottom) lenses each, in general (on the left) and in flat (on the right) cosmological models. Thick bars on the co-ordinate axes and contour levels on the planes represent, respectively, the $95 \%$ confidence intervals and the $68 \%$ and $95 \%$ confidence regions for the cosmological parameters. Crosses show the position of the true parameters: $\left(\Omega_{\mathrm{m}}, \Omega_{\Lambda}\right)=(0.3,0.7)$.

the following we will consider only data for which refined lens models are available.) The first estimator $\left(\theta_{1}\right)$ is given by the semi-distance between the two images, and the second one $\left(\theta_{2}\right)$ by the semi-sum of the distances of the two images from the
Table 3. Intervals at $95 \%$ confidence level for the matter and the darkenergy density parameters in general (second and third columns) and flat (fourth and fifth columns) cosmology.

\begin{tabular}{ccccc}
\hline \hline & $\Omega_{\mathrm{m}}$ & $\Omega_{\Lambda}$ & $\Omega_{\mathrm{m}}{ }^{a}$ & $\Omega_{\Lambda}{ }^{a}$ \\
\hline$N=100$ & {$[0.00,1.12]$} & {$[0.52,0.79]$} & {$[0.27,0.45]$} & {$[0.55,0.73]$} \\
$N=200$ & {$[0.00,0.80]$} & {$[0.54,0.74]$} & {$[0.29,0.42]$} & {$[0.58,0.71]$} \\
\hline
\end{tabular}

${ }^{a}$ Flat cosmology: $\Omega_{\mathrm{m}}+\Omega_{\Lambda}=1$.

Notes. These intervals are obtained by excluding from the $2000 \chi^{2}$ minimizations the 50 smallest and the 50 largest values for $\Omega_{\mathrm{m}}$ and $\Omega_{\Lambda}$. The simulated measurement uncertainties are $5 \%$ of the true values for both the Einstein angle and the central velocity dispersion. Samples of 100 and 200 lenses are considered.

center of the lens. The triangular inequality ensures that $\theta_{2}$ is always greater than $\theta_{1}$. We remark that the first quantity is easy to measure, even when the lens (mass) center is not known a priori; anyway, in most cases the lens center should be identified with the galaxy luminosity centroid. The two estimators coincide when a single axisymmetric model is considered, but they differ when some ellipticity for the lens or an external shear component is present. In Fig. 5 and Table 4 we illustrate the statistical properties of $\theta_{1}$ and $\theta_{2}$, obtained from simulations in which the physical variables were selected as described in the previous section. The consistency test and the values of bias and mean squared error (MSE) (for definitions see Cowan 1998) favour $\theta_{2}$ as the better estimator for $\theta_{\mathrm{E}}$.

\subsection{The velocity dispersion}

The velocity dispersion of stars in galaxies is a well-defined dynamical quantity (see Bertin 2000). The expression "velocity dispersion" is also used in lensing studies to refer to a parameter of the $1 / r^{2}$ density distribution characteristic of the 

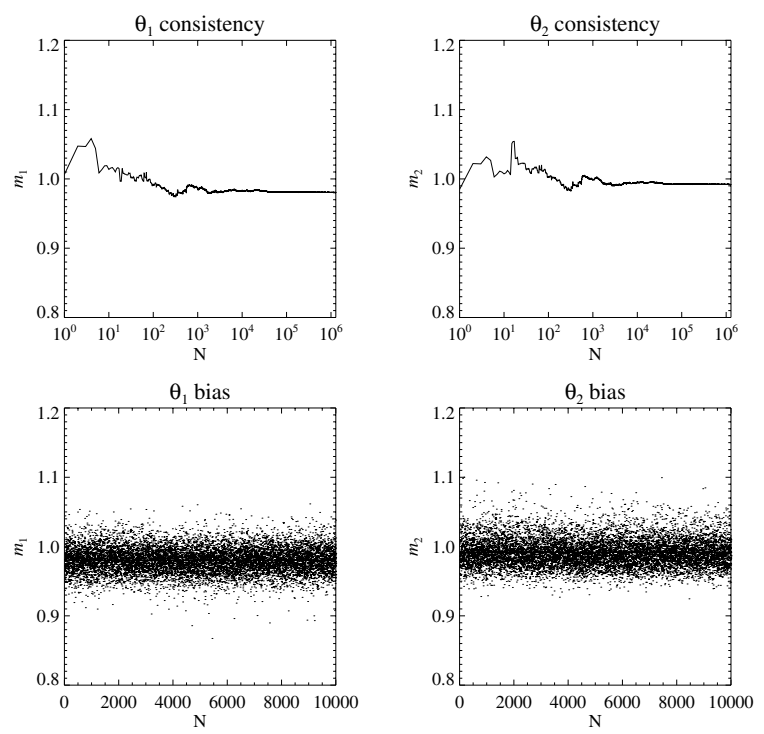

Fig. 5. Consistency and bias tests for the $\theta_{\mathrm{E}}$ estimators. Top: angular coefficients $\left(m_{1}\right.$ and $\left.m_{2}\right)$ of the linear fits of the true Einstein angle versus the estimated value from $\theta_{1}$ (on the left) and $\theta_{2}$ (on the right), for an increasing number of lenses. Bottom: angular coefficients of the linear fits of the true Einstein angle versus the estimated value from $\theta_{1}$ (on the left) and $\theta_{2}$ (on the right) for 10000 samples with 100 lenses each. Solid lines show the mean values.

Table 4. Statistics of the two estimators of the Einstein angle.

\begin{tabular}{ccc}
\hline \hline & Bias & MSE \\
\hline$\theta_{1}$ & -0.020 & 0.0008 \\
$\theta_{2}$ & -0.008 & 0.0005 \\
\hline
\end{tabular}

isothermal sphere. In fact, the measured total mass of a lens within the Einstein angle can be easily converted into an effective velocity dispersion, given the relation between $\theta_{\mathrm{E}}$ and $\sigma_{\mathrm{SIS}}$ of Eq. (1). As already mentioned in Sect. 2, a one-component isothermal model has proved to be an adequate description for the total density distribution in elliptical galaxies, as far as lensing is concerned. On the other hand, dynamical modeling requires two-component (luminous+dark) models in order to find valid agreement with the observations; in principle, the velocity dispersion of the stellar component might be different from that of the dark component. Here we wish to compare the central value of the stellar velocity dispersion to the velocity dispersion of a one-component isothermal model supposed to represent the total (stellar and dark) matter distribution. We will find that a good estimator of the one-component velocity dispersion of an isothermal model $\left(\sigma_{\text {SIS }}\right)$ is indeed the stellar central velocity dispersion $\left(\sigma_{0}\right)$. This step is essential in order to apply the method proposed in this paper.

We have considered eight bright, nearly round, early-type galaxies, which were modeled as described in Bertin et al. (1992) and Saglia et al. (1992). We assumed that the total mass of each galaxy could be described in terms of a singular isothermal sphere and calculated $\sigma_{\text {SIS }}$ starting from the best-fit twocomponent models of Saglia et al. (1992). Then, using the kinematical data reported in Davies \& Birkinshaw (1988) and Franx et al. (1989), we estimated $\sigma_{0}$ for the same galaxy sample. The results are summarized in Table 5; the ratio of the two velocities ( $\left.q=\sigma_{0} / \sigma_{\text {SIS }}\right)$ is displayed in Fig. 6 . The uncertainty on $\sigma_{0}$ is just the rms scatter of the velocity dispersions measured at different slit position angles, so it is probably an underestimate of the real
Table 5. Velocity dispersions and dark matter fraction for a sample of eight bright, nearly round, early-type galaxies.

\begin{tabular}{ccccc}
\hline \hline Object & NGC 1404 & NGC 1549 & NGC 3379 & NGC 4278 \\
\hline$\sigma_{0}$ & $245 \pm 7$ & $198 \pm 6$ & $219 \pm 13$ & $228 \pm 9$ \\
$\sigma_{\text {SIS }}$ & 259 & 196 & 212 & 232 \\
$f_{\text {DM }}$ & 0.58 & 0.59 & 0.40 & 0.18 \\
\hline Object & NGC 4374 & NGC 4472 & NGC 4486 & NGC 4636 \\
\hline$\sigma_{0}$ & $291 \pm 6$ & $292 \pm 7$ & $311 \pm 3$ & $206 \pm 8$ \\
$\sigma_{\text {SIS }}$ & 280 & 302 & 286 & 213 \\
$f_{\text {DM }}$ & 0.59 & 0.33 & 0.34 & 0.27 \\
\hline
\end{tabular}

Notes. Stellar central velocity dispersion $\left(\sigma_{0}\right)$, one-component velocity dispersion of the singular isothermal sphere $\left(\sigma_{\mathrm{SIS}}\right)$ that best fits the photometry and the kinematics, and dark matter mass fraction inside $R_{\mathrm{e}}\left(f_{\mathrm{DM}}\right)$.

References. Davies \& Birkinshaw (1988); Franx et al. (1989); Saglia et al. (1992).

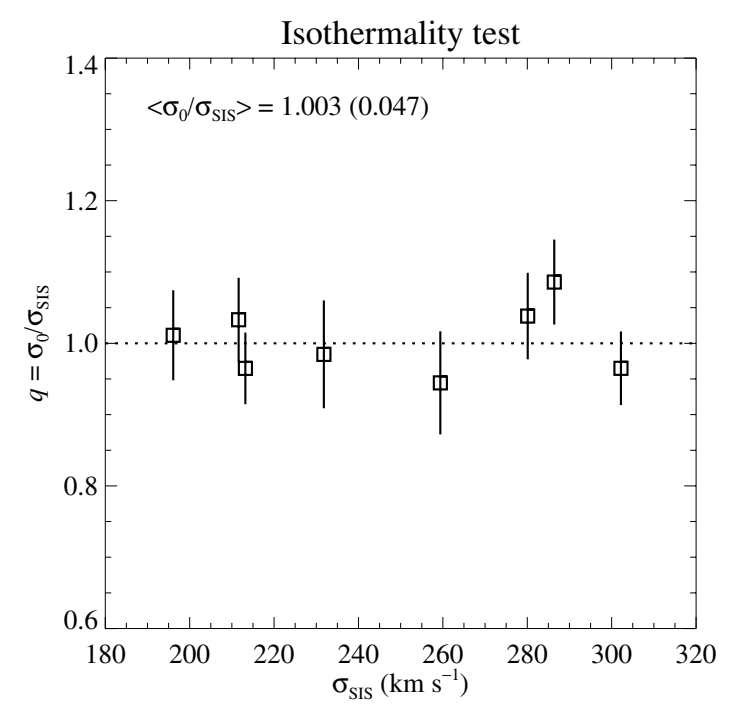

Fig. 6. Ratio $(q)$ of the stellar central velocity dispersion $\left(\sigma_{0}\right)$ to the onecomponent velocity dispersion of the singular isothermal sphere $\left(\sigma_{\mathrm{SIS}}\right)$ that best fits the photometry and the kinematics for a sample of eight bright, nearly round, early-type galaxies.

value. In addition to this, a minimum $5 \%$ error on the $\sigma_{\text {SIS }}$ was assumed in order to obtain the error bars of Fig. 6. We notice that $q$ has an average value very close to unity $(1.003 \pm 0.017)$ and an intrinsic rms scatter remarkably small (0.047). Therefore, we infer that $\sigma_{0}$, which is related to the stellar component alone, is a good diagnostic of $\sigma_{\text {SIS }}$, a tracer of the total (stellar and dark) matter distribution. Moreover, the ratio $q$ does not show any significant dependence either on the $\sigma_{\text {SIS }}$ value, as can be seen in Fig. 6 , or on the dark matter fraction $f_{\mathrm{DM}}$ inside $R_{\mathrm{e}}$, as reported in Table 5. This fact suggests the existence of an efficient mechanism of coupling between stellar and dark mass ("conspiracy"). In addition, we also remark that $q$ is not influenced by a particular choice of the cosmological parameter values, because the galaxy set is placed in the nearby Universe.

A larger sample (the SLACS sample, described in the following section) of more distant early-type galaxies, but with essentially the same range of velocity dispersion, was studied similarly by Treu et al. (2006). There they estimated the isothermal velocity dispersion inside the Einstein radius $R_{\mathrm{E}}$ from the best lensing model and their results about the value and intrinsic scatter of $q$ are consistent with what we found. In conclusion, since we demonstrated that $\sigma_{0}$ is a good estimator of $\sigma_{\text {SIS }}$, we argue 
Table 6. The lens samples of the SLACS (1-15) and LSD (16-20) surveys.

\begin{tabular}{cccccc}
\hline \hline$\#$ & $z_{\mathrm{l}}$ & $z_{\mathrm{s}}$ & $\theta_{\mathrm{E}}\left(^{\prime \prime}\right)$ & $\sigma_{0}\left(\mathrm{~km} \mathrm{~s}^{-1}\right)$ & $R_{\mathrm{e}}\left(^{\prime \prime}\right)$ \\
\hline 1 & 0.1955 & 0.6322 & 1.47 & $282 \pm 11$ & $2.38 \pm 0.02$ \\
2 & 0.3317 & 0.5235 & 1.15 & $349 \pm 24$ & $3.37 \pm 0.22$ \\
3 & 0.3223 & 0.5812 & 1.03 & $326 \pm 16$ & $3.26 \pm 0.13$ \\
4 & 0.1642 & 0.3240 & 1.61 & $325 \pm 12$ & $4.81 \pm 0.02$ \\
5 & 0.2405 & 0.4700 & 1.32 & $318 \pm 17$ & $2.60 \pm 0.03$ \\
6 & 0.1260 & 0.5349 & 1.00 & $229 \pm 13$ & $1.82 \pm 0.05$ \\
7 & 0.2318 & 0.7950 & 1.15 & $274 \pm 15$ & $1.77 \pm 0.01$ \\
8 & 0.0808 & 0.7115 & 0.85 & $195 \pm 10$ & $1.23 \pm 0.01$ \\
9 & 0.2046 & 0.4814 & 1.39 & $290 \pm 16$ & $3.14 \pm 0.02$ \\
10 & 0.0629 & 0.5352 & 1.04 & $206 \pm 5$ & $2.60 \pm 0.10$ \\
11 & 0.2076 & 0.5241 & 1.21 & $295 \pm 13$ & $2.14 \pm 0.02$ \\
12 & 0.2479 & 0.7933 & 1.81 & $279 \pm 17$ & $2.02 \pm 0.02$ \\
13 & 0.2285 & 0.4635 & 1.25 & $305 \pm 19$ & $1.80 \pm 0.01$ \\
14 & 0.1553 & 0.5170 & 1.64 & $271 \pm 16$ & $4.20 \pm 0.04$ \\
15 & 0.0819 & 0.5324 & 1.57 & $245 \pm 7$ & $4.47 \pm 0.01$ \\
\hline 16 & 0.485 & 3.595 & 1.34 & $229 \pm 15$ & $0.82 \pm 0.12$ \\
17 & 0.938 & 2.941 & 1.24 & $251 \pm 19$ & $1.60 \pm 0.15$ \\
18 & 0.810 & 3.399 & 1.41 & $224 \pm 15$ & $1.06 \pm 0.08$ \\
19 & 0.497 & 2.092 & 0.36 & $116 \pm 10$ & $0.41 \pm 0.04$ \\
20 & 1.004 & 3.263 & 1.56 & $328 \pm 32$ & $0.31 \pm 0.06$ \\
\hline
\end{tabular}

References. Treu et al. (2002, 2003, 2004, 2006); Koopmans et al. (2002, 2003a, 2006).

that the former quantity can be substituted in Eq. (1) to get the cosmology-dependent relation given by Eq. (2).

\section{The SLACS and LSD samples}

As an application of our technique, we considered the SLACS (Sloan Lens ACS) Survey data set presented in Bolton et al. (2006), Treu et al. (2006), and Koopmans et al. (2006) (see Table 6). The sample is composed of fifteen massive, early-type, lens galaxies at redshifts $z_{1}$ ranging from 0.06 to 0.33 and background sources with a redshift range of $z_{\mathrm{s}}$ from 0.32 to 0.80 . Detailed gravitational lensing models on the HST/ACS images were developed in order to measure the total mass inside the Einstein angle, i.e. $\theta_{\mathrm{E}}$ for an isothermal model, to a less than a few percent accuracy; from the same images also the values of the galaxy effective radii $\left(R_{\mathrm{e}}\right)$ were derived. Furthermore, $\sigma_{0}$ and its uncertainty (average value of $5.0 \%$ ) were obtained from the Sloan Digital Sky Survey Database. Afterwards, we studied a second data set, adding to the previous one five additional field elliptical galaxies from the LSD (Lenses Structure and Dynamics) Survey (Koopmans \& Treu 2002, 2003a; Treu \& Koopmans 2002, 2003, 2004) (see Table 6). These lens and source galaxies are at higher redshifts $\left(z_{1} \approx 0.5-1.0, z_{\mathrm{s}} \approx\right.$ 2.0-3.6), thus it follows from Sect. 2 that the dependence of $r$ on the cosmological parameters should be easier to test. On the other hand, these galaxies have higher central velocity dispersion errors, so that the mean uncertainty on $\sigma_{0}$ rises now to $5.7 \%$.

At first, we checked on the two samples the plausibility of the hypothesis of homologous total density distribution, without any assumptions on the values of the cosmological parameters. In order to do so, we took into account the following relation (Koopmans 2005)

$\frac{c^{2}}{4 \pi} \frac{\theta_{\mathrm{E}}}{\sigma_{0}^{2}}=r\left(z_{\mathrm{l}}, z_{\mathrm{s}} ; \Omega_{\mathrm{m}}, \Omega_{\Lambda}\right)\left(\frac{8 \theta_{\mathrm{E}}}{R_{\mathrm{e}}}\right)^{2-\gamma} \frac{\gamma(3-\gamma)}{2}$,

which extends Eq. (2), considering a more general power law model for the total density profile $\left(\rho \propto 1 / r^{\gamma}\right)$ of the lens galaxies
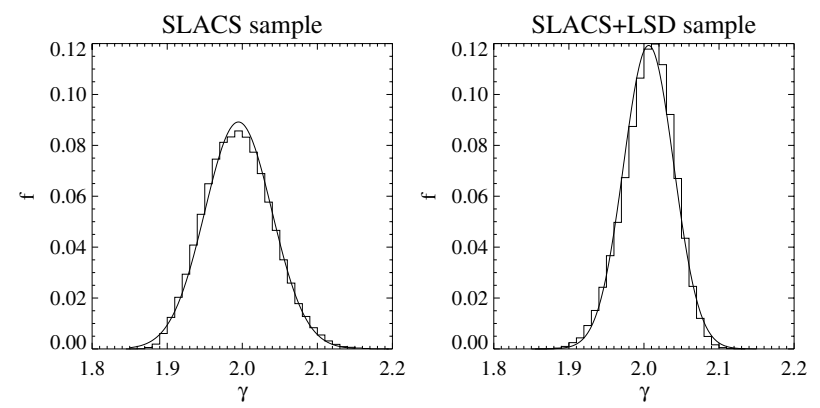

Fig. 7. Probability density function $(f)$, marginalized over the values of the cosmological parameters, of the exponent $(\gamma)$ which characterizes the total density profile of the lens galaxies. The probability distribution with the approximate normal distribution for the SLACS (on the left) and for the SLACS+LSD (on the right) samples are obtained through MCMC methods.

Table 7. Mean value and standard deviation of the parameter $\gamma$ for the SLACS and SLACS+LSD samples.

\begin{tabular}{ccc}
\hline \hline & SLACS & SLACS+LSD \\
\hline$\gamma$ & $1.99 \pm 0.04$ & $2.01 \pm 0.03$ \\
\hline
\end{tabular}

(the isothermal case is retrieved setting $\gamma$ equal to 2). The parameter $\gamma$ was assumed to be the same for all the lenses and we used Markov chain Monte Carlo (MCMC) methods (with $5 \times 10^{5}$ steps for each chain) to sample its probability density distribution. The marginalized probability density function $(f)$ with the approximate normal distribution, the mean value, and the standard deviation of $\gamma$ for the two SLACS and SLACS+LSD samples are shown in Fig. 7 and Table 7. From these results, we can state that the total density profile of the lens ellipticals is indeed well approximated by an isothermal distribution ( $\gamma$ equal to 2 ), independentely on the adopted cosmological model.

Starting from here, we evaluated the observational angular diameter distance ratio of Eq. (2) and its uncertainty, assuming a reasonable $5 \%$ error on $\theta_{\mathrm{E}}$. Hence, we used the $\chi^{2}$ statistics reported in Eq. (3) to gain information about the cosmological parameters.

The results are plotted in Figs. 8 and 9. Here we show the intervals at the $95 \%$ confidence level for the dark-energy density parameter in a flat cosmological model, and the $68 \%, 95 \%$, and $99 \%$ confidence regions in the parameter space $\left(\Omega_{\mathrm{m}}, \Omega_{\Lambda}\right)$ for general cosmological models. In a flat geometry, at $95 \% \mathrm{CL}$, $\Omega_{\Lambda}$ is found to be smaller than 0.80 from the SLACS sample, and greater than 0.16 from the SLACS+LSD sample. In addition, without any assumptions on the cosmological parameters, the SLACS+LSD sample rules out, at greater than $99 \%$ CL, cosmological models with dark-energy density parameter smaller than 0.4 (as shown in the bottom right panel of Fig. 9). From a comparison of Figs. 8 and 9, it turns out that the larger set of galaxies with more distant lenses shifts the minimum $\chi^{2}$ towards higher values of $\Omega_{\Lambda}$ and that a much bigger region of values for the cosmological parameters is excluded, at the same confidence level.

The small number of lenses in the two samples is the primary reason that prevents us from reaching higher precision results. We note that the current concordance measurement of $\Omega_{\Lambda}$ suggests a value between 0.7 and 0.8 and this range is included in the $95 \%$ confidence intervals and in the $99 \%$ confidence regions for both the SLACS and the SLACS+LSD samples. 

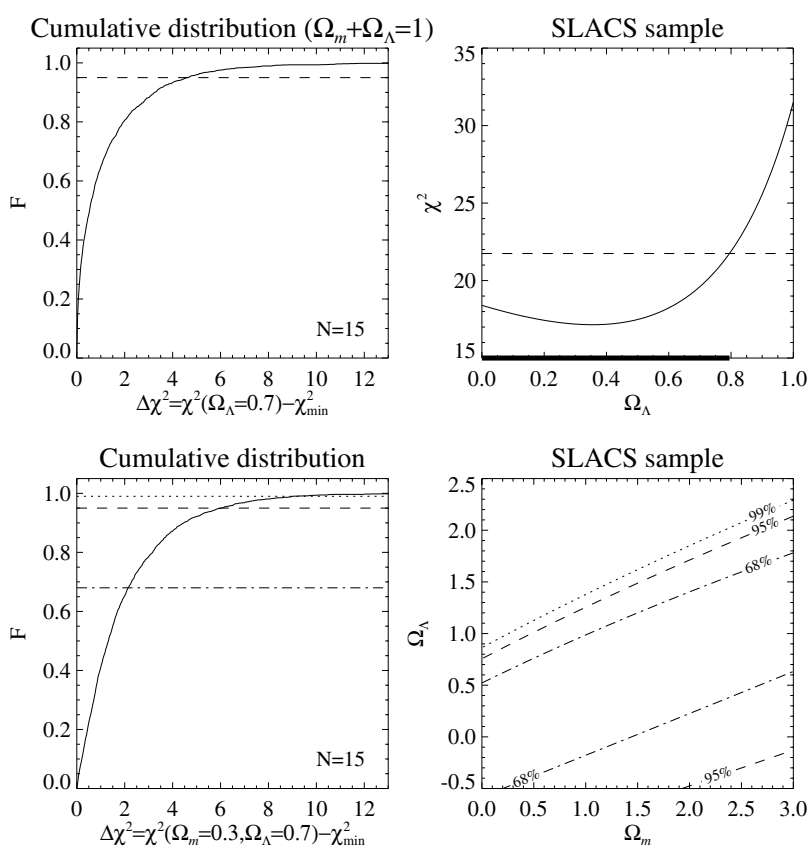

Fig. 8. Results from the SLACS sample. Top left: cumulative distribution $(F)$ of the distance between the $\chi^{2}$ in the true $\left(\Omega_{\Lambda}=0.7\right)$ and the best flat cosmological model from 2000 simulations with 15 lenses each. The dashed line gives the $95 \%$ confidence level. Top right: the $\chi^{2}$ curve for the SLACS sample in a flat cosmology. The dashed line corresponds to the value of $\Delta \chi^{2}$ of the previous panel and the thick bar on the abscissa axis shows the $95 \%$ confidence level interval for $\Omega_{\Lambda}$. Bottom left: cumulative distribution $(F)$ of the distance between the $\chi^{2}$ in the true $\left(\Omega_{\mathrm{m}}, \Omega_{\Lambda}\right)=(0.3,0.7)$ and the best cosmological model from 2000 simulations with 15 lenses each. The dashed-dotted, dashed, and dotted lines give, respectively, the $68 \%, 95 \%$, and $99 \%$ confidence levels. Bottom right: the $\chi^{2}$ contour plot for the SLACS sample; the levels correspond to the values of $\Delta \chi^{2}$ of the previous panel. The dasheddotted, dashed, and dotted lines show, respectively, the 68\%, 95\%, and $99 \%$ confidence regions for $\Omega_{\mathrm{m}}$ and $\Omega_{\Lambda}$.

\section{Conclusions}

Lensing studies have been recognized to be very valuable in providing a testing ground for theories of formation and evolution of early-type galaxies (Rusin \& Kochanek 2003; Koopmans et al. 2006). In these studies the structure of the lens galaxies can be investigated, once a specific cosmological model is adopted. From a complementary perspective, a measurement of the cosmological parameters can in principle be accomplished by assuming some empirically justified general properties for a sample of lenses. In this paper, we have shown that indeed, starting from the $1 / r^{2}$ paradigm for the total density profile of elliptical galaxies, the cosmological parameters $\Omega_{\mathrm{m}}$ and $\Omega_{\Lambda}$ can be estimated. The adopted paradigm has been validated by stellar dynamics (e.g., Gerhard et al. 2001), strong gravitational lensing (e.g., Rusin et al. 2003), and dynamical studies based on several other tracers: globular clusters and planetary nebulae (e.g., Peng et al. 2004), X-ray halos (e.g., Humphrey et al. 2006), and HI disks and rings (e.g., Franx et al. 1994).

In detail, the main results of this paper can be summarized as follows:

- We have developed a new method to investigate the geometry of the Universe by combining measurements of strong gravitational lensing and stellar dynamics in a sample of elliptical galaxies. The basic idea is to compare lensing and dynamical mass estimates in order to find an observable
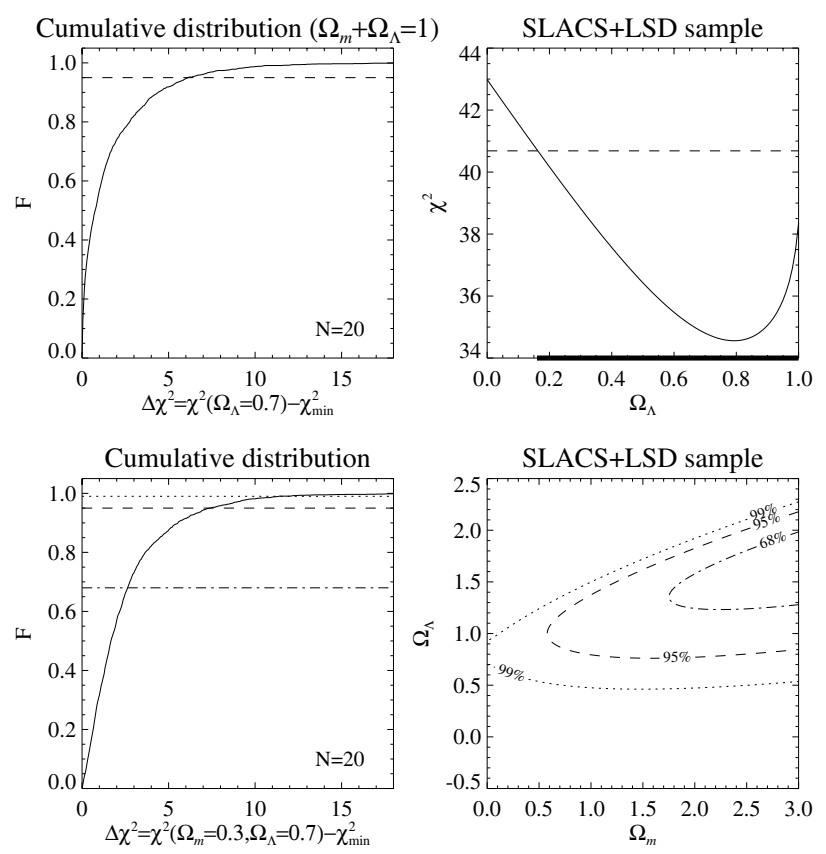

Fig. 9. Results from the SLACS+LSD sample. Top left: cumulative distribution $(F)$ of the distance between the $\chi^{2}$ in the true $\left(\Omega_{\Lambda}=0.7\right)$ and the best flat cosmological model from 2000 simulations with 20 lenses each. The dashed line gives the $95 \%$ confidence level. Top right: the $\chi^{2}$ curve for the SLACS+LSD sample in a flat cosmology. The dashed line corresponds to the value of $\Delta \chi^{2}$ of the previous panel and the thick bar shows the $95 \%$ confidence level interval for $\Omega_{\Lambda}$. Bottom left: cumulative distribution $(F)$ of the distance between the $\chi^{2}$ in the true $\left(\Omega_{\mathrm{m}}, \Omega_{\Lambda}\right)=(0.3,0.7)$ and the best cosmological model from 2000 simulations with 20 lenses each. The dashed-dotted, dashed, and dotted lines give, respectively, the $68 \%, 95 \%$, and $99 \%$ confidence levels. Bottom right: the $\chi^{2}$ contour plot for the SLACS+LSD sample; the levels correspond to the values of $\Delta \chi^{2}$ of the previous panel. The dashed-dotted, dashed, and dotted lines show, respectively, the 68\%, 95\%, and 99\% confidence regions for $\Omega_{\mathrm{m}}$ and $\Omega_{\Lambda}$.

cosmology-dependent relation. For this purpose, it is necessary to model the total (luminous and dark) density profile inside the Einstein radius of the ellipticals under investigation. Here, we have assumed a one-component $1 / r^{2}$ density profile.

- We have performed a feasibility study of our technique by simulating measurements of the cosmological parameters. We have studied the precision of the method, which depends on the level of uncertainty with which the Einstein angle and the central stellar velocity dispersion are known. Hence, we have demonstrated that the current uncertainty with which these quantities can be measured is sufficiently small to obtain precise information about cosmology, once a statistically significant sample of lenses is available. In particular, the method has been shown to be best suited to measure $\Omega_{\Lambda}$.

- Then, we have studied the relation between the stellar kinematics and the velocity parameter of the one-component $1 / r^{2}$ (isothermal) model for the total density distribution in ellipticals. For a sample of eight bright, round, and nearby earlytype galaxies, we have shown that the stellar central velocity dispersion is a good tracer of the velocity dispersion characterizing the one-component model.

- We have applied the proposed method to the SLACS Survey data set. This sample is composed of fifteen massive earlytype galaxies at intermediate redshift acting as lenses. In a flat cosmology this method leads to a value of $\Omega_{\Lambda}$ lower 
than 0.80 at $95 \%$ CL. By including five more distant lenses from the LSD Survey, at the same confidence level and in the same flatness hypothesis, $\Omega_{\Lambda}$ has been measured to be greater than 0.16 . This latter sample ruled out, at greater than $99 \% \mathrm{CL}$, general cosmological models with values of $\Omega_{\Lambda}$ smaller than 0.4 .

- Finally, we conclude that future surveys expected to identify a large number of lenses (one or two hundred) will allow this method to measure the values of the cosmological parameters with a precision comparable to that of other standard techniques. This will be a new and important test for the $\Lambda C D M$ concordance model.

Acknowledgements. This work was partly supported by the Italian MiUR (PRIN 2004). G.B. and C.G. acknowledge the support and hospitality of the Kavli Institute for Theoretical Physics at the University of California, Santa Barbara.

\section{References}

Bartelmann, M., \& Schneider, P. 1999, A\&A, 345, 17

Bartelmann, M., \& Weiss, A. 1994, A\&A, 287, 1

Bartelmann, M., Huss, A., Colberg, J., Jenkins, A., \& Pearce, F. 1998, A\&A, 330,1

Bennett, C. L., Halpern, M., Hinshaw, G., et al. 2003, ApJS, 148, 1

Bertin, G. 2000, Dynamics of Galaxies (Cambridge University Press)

Bertin, G., Ciotti, L., \& Del Principe, M. 2002, A\&A, 386, 149

Bertin, G., Saglia, R. P., \& Stiavelli, M. 1992, ApJ, 384, 423

Bolton, A. S., Burles, S., Koopmans, L. V. E., Treu, T., \& Moustakas, L. A. 2006, ApJ, 638, 703

Browne, I. W. A., Wilkinson, P. N., Jackson, N. J. F., et al. 2003, MNRAS, 341, 13

Burles, S., Nollett, K. M., \& Turner, M. S. 2001, ApJ, 552, 1

Cole, S., Percival, W. J., Peacock, J. A., et al. 2005, MNRAS, 362, 505

Cowan, G. 1998, Statistical Data Analysis (Clarendon Press, Oxford)

Cyburt, R. H., Ellis, J., Fields, B. D., \& Olive, K. A. 2003, Phys. Rev. D, 67, 103521

Dalal, N., Holder, G., \& Hennawi, J. F. 2004, ApJ, 609, 50

Davies, R. L., \& Birkinshaw, M. 1988, ApJS, 68, 409

Franx, M., Illingworth, G., \& Heckman, T. 1989, ApJ, 344, 613

Franx, M., van Gorkom, J. H., \& de Zeeuw, T. 1994, ApJ, 436, 642
Freedman, W. L., Madore, B. F., Gibson, B. K., et al. 2001, ApJ, 553, 47 Gerhard, O., Kronawitter, A., Saglia, R. P., \& Bender, R. 2001, AJ, 121, 1936 Humphrey, O., Buote, D. A., Gastaldello, F., et al. 2006, ApJ, 646, 899 Jain, B., \& Taylor, A. 2003, Phys. Rev. Lett., 91, 141302

Kochanek, C. S. 1992, ApJ, 384, 1

Kochanek, C. S. 1993, ApJ, 419, 12

Kochanek, C. S. 1994, ApJ, 436, 56

Kochanek, C. S. 1996, ApJ, 473, 595

Koopmans, L. V. E. 2005 [arXiv:astro-ph/0511121]

Koopmans, L. V. E., \& Treu, T. 2002, ApJ, 568, 5

Koopmans, L. V. E., \& Treu, T. 2003a, ApJ, 583, 606

Koopmans, L. V. E., Treu, T., Fassnacht, C. D., Blandford, R. D., \& Surpi, G. 2003b, ApJ, 599, 70

Koopmans, L. V. E., Treu, T., Bolton, A. S., Burles, S., \& Moustakas, L. A. 2006, ApJ, 649, 599

Kormann, R., Schneider, P., \& Bartelmann, M. 1994, A\&A, 284, 285

Link, R., \& Pierce, M. J. 1998, ApJ, 502, 63

Lombardi, M., \& Bertin, G. 1999, A\&A, 342, 337

Mitchell, J. L., Keeton, C. R., Frieman, J. A., \& Sheth, R. K. 2005, ApJ, 622, 81

Mörtsell, E., \& Sunesson, C. 2006, JCAP, 01, 012

Myers, S. T., Jackson, N. J., Browne, I. W. A., et al. 2003, MNRAS, 341, 1

Myungshin, I., Griffiths, R. E., \& Ratnatunga, K. U. 1997, ApJ, 475, 457

Peng, E. W., Ford, H. C., \& Freeman, K. C. 2004, ApJ, 602, 705

Perlmutter, S., Aldering, G., Goldhaber, G., et al. 1999, ApJ, 517, 565

Refregier, A. 2003, ARA\&A, 41, 645

Refsdal, S. 1964, MNRAS, 128, 307

Riess, A. G., Filippenko, A. V., Challis, P., et al. 1998, AJ, 116, 1009

Riess, A. G., Strolger, L.-G., Tonry, J., et al. 2004, ApJ, 607, 665

Rusin, D., \& Kochanek, C. S. 2005, ApJ, 623, 666

Rusin, D., Kochanek, C. S., \& Keeton, C. R. 2003, ApJ, 595, 29

Saglia, R. P., Bertin, G., \& Stiavelli, M. 1992, ApJ, 384, 433

Schneider, P., Ehlers, J., \& Falco, E. E. 1992, Gravitational Lenses (New York: Springer-Verlag)

Soucail, G., Kneib, J.-P., \& Golse, G. 2004, A\&A, 417, 33

Spergel, D. N., Verde, L., Peiris, H. V., et al. 2003, ApJS, 148, 175

Spergel, D. N., Bean, R., Doré, O., et al. 2007, ApJS, 170, 377

Tegmark, M., Strauss, M. A., Blanton, M. R., et al. 2004, Phys. Rev. D, 69, 10350

Treu, T., \& Koopmans, L. V. E. 2002, ApJ, 575, 87

Treu, T., \& Koopmans, L. V. E. 2003, MNRAS, 343, 29

Treu, T., \& Koopmans, L. V. E. 2004, ApJ, 611, 739

Treu, T., Stiavelli, M., Casertano, S., Møller, P., \& Bertin, G. 2002, ApJ, 564, 13

Treu, T., Koopmans, L. V. E., Bolton, A. S., Burles, S., \& Moustakas, L. A. 2006, ApJ, 640, 662

Witt, H. J., \& Mao, S. 1997, MNRAS, 291, 211 